\title{
Triazolo[1,5-c]quinazolines. Synthesis, antimicrobial and antifungal activity [quinazolin-4(3H)-ylidene]hydrazides of carboxylic acids (Message 1)
}

\author{
K. P. Schabelnyk, S. V. Kholodnyak, G. G. Berest, S. I. Kovalenko, N. M. Polishchuk, \\ O. M. Kamyshnyi
}

Zaporizhzhia State Medical University, Ukraine

The modern standards and algorithms of antimicrobial therapy, that ensure a high level of the treatment's quality and prevention of the most infectious-inflammatory diseases, provide wide using of chemotherapeutic agents. However, the modern medicines do not always have satisfactory chemotherapeutic and pharmacological properties; this fact is most often associated with the resistance of microorganisms to them. That is why the creation of the new chemotherapeutic drugs by natural antibiotics' chemical and microbiological modification, the chemical synthesis of the new substances among the various classes of organic compounds is a topical problem.

The aim of the study is the developing of simple and accessible methods for the synthesis of new [quinazoline-4(3H)-ylidene] hydrazides of cycloalkyl-(hetaryl) carboxylic acids, analysis of their physical-chemical properties, antimicrobial and antifungal activity.

Materials and methods. The study of the antimicrobial activity of synthesized compounds was carried out by the method of twofold serial dilutions in the Mueller Hinton broth (for strains Staphylococcus aureus ATCC 25923, Escherichia coli ATCC 25922, Pseudomonas aeruginosa ATCC 27853) and in the Saburo broth (for Candida albicans ATCC 885-653). Minimal inhibitory concentration, minimal bactericidal and fungicidal concentration were defined.

Results. It was found that synthesized compounds show antimicrobial and antifungal activity to researched strains.

Conclusions. There have been considered a number of preparative methods for the synthesis of [quinazoline-4(3H)-ylidene] hydrazides of cycloalkyl-(hetaryl)carboxylic acids, which are significant as chemical reagents for the preparation of condensed heterocyclic and biologically active compounds. The structure and individuality have been proved by elementary analysis and physical-chemical methods ('H NMR-spectroscopy, HPLC/MS). The antimicrobial analysis shows that N'-[quinazoline-4(3H)ylidene]-2(3)-heteril hydrazides containing a pyridinecarboxylic acid residue in the molecule have the highest activity, which exceeds the activity of the reference standard "Trimethoprim". N"-(6-bromohinazolin-4(3H)-ylidene)benzofuryl-2-carbohydrazide demonstrates the highest antifungal activity, which is comparable with the reference standard "Ketoconazole".
Тріазоло[1,5-с]хіназоліни. Синтез, протимікробна та протигрибкова активність [хіназолін-4(3Н)-іліден]гідразидів карбонових кислот (Повідоммення 1)

\section{К. П. Шабельник, С. В. Холодняк, Г. Г. Берест, С. І. Коваленко, Н. М. Поліщук, О. М. Камишний}

Сучасні стандарти та алгоритми антимікробної терапії, що забезпечують високий рівень якості лікування та профілактики більшості захворювань інфекційно-запального характеру, передбачають широке використання хіміотерапевтичних засобів. Однак сучасні лікарські засоби не завжди мають задовільні хіміотерапевтичні та фрармакологічні властивості, що найчастіше пов'язано з резистентністю мікроорганізмів до них. Саме тому створення нових хіміотерапевтичних препаратів шляхом хімічної та мікробіологічної модиффікації природних антибіотиків, хімічного синтезу нових речовин серед різних класів органічних сполук є актуальною проблемою.

Мета роботи - розроблення простих і доступних методів синтезу нових [хіназолін-4(3Н)-іліден]гідразидів циклоалкіл-(гетарил-)карбонових кислот, вивчення їхніх фізико-хімічних властивостей, дослідження протимікробної та протигрибкової активності.

Матеріали та методи. Протимікробну активність синтезованих хімічних сполук виконали методом дворазових серійних розведень у бульйоні Мюллера-Хінтона (для штамів Staphylococcus aureus ATCC 25923, Escherichia coli ATCC 25922, Pseudomonas aeruginosa ATCC 27853) і в бульйоні Сабуро (для Candida albicans ATCC 885-653). Визначали МІК (мінімальна інгібуюча концентрація), МБцК і МФцК (мінімальна бактерицидна і фунгіцидна концентрації відповідно).

Результати. Встановлено, що синтезовані сполуки проявляють протимікробну та протигрибкову активність до досліджуваних штамів.

Висновки. Розглянули препаративні методи синтезу [хіназолін-4(3Н)-іліден]гідразидів циклоалкіл-(гетарил-)карбонових кислот, які є цінними хімічними реагентами для отримання конденсованих гетероциклічних і біологічно активних сполук. Будова й індивідуальність підтверджені за допомогою елементного аналізу та фізико-хімічних методів ('H ЯМР-спектроскопія, хромато-мас-спектрометрія). Аналіз результатів мікробіологічного дослідження показує, що серед синтезованих сполук найбільшу протимікробну активність мають $N^{\prime}$-[хіназолін-4(3H)-іліден]-2(3)-гетерил-гідразиди, які містять у молекулі залишок піридинкарбонової кислоти, що перевищує активність еталону порівняння триметоприму. Найбільшу протигрибкову активність проявляє $N^{\prime}$-(6-бромохіназолін-4(3Н)-іліден)бензофуро-2-карбогідразид, який можна порівняти з еталоном порівняння кетоконазолом.
Key words:

N'-(quinazolin4(3H)-ylidene) carbohydrazides, synthesis, antimicrobial agents, antifungal agents.

Zaporozhye medical journal 2018; 20 (3), 425-431 DOI: 10.14739/2310-1210 2018.3.130525

E-mail: kshabelnik@gmail.com

Ключові слова: [хіназолін4(3Н)-іміден] гіАразиди, синтез, протимікробна активність, протигрибкова активність.

Запорізький медичний журнал. - 2018. T. 20, № 3(108). -

C. 425-431 
Ключевые слова: [хиназомин-4(3Н)-илиАен] гиАразиАЫ, Синтез, противомикробная активность, противогрибковая активность.

Запорожский медицинский журнах. - 2018. T. 20, № 3(108). C. 425-431

\title{
Триазоло[1,5-с]хиназолины. Синтез, противомикробная и противогрибковая активность [хиназолин-4(3Н)-илиден]гидразидов карбоновых кислот (Сообщение 1)
}

\author{
К. П. Шабельник, С. В. Холодняк, Г. Г. Берест, С. И. Коваленко, Н. М. Полищук, А. М. Камышный
}

Современные стандарты и алгоритмы антимикробной терапии, обеспечивающие высокий уровень качества лечения и профилактики большинства заболеваний инфекционно-воспалительного характера, предусматривают широкое использование химиотерапевтических средств. Однако современные лекарственные средства не всегда имеют удовлетворительные химиотерапевтические и фармакологические свойства, что чаще всего связано с резистентностью микроорганизмов к ним. Именно поэтому создание новых химиотерапевтических препаратов путем химической и микробиологической модификации природных антибиотиков, химического синтеза новых веществ среди различных классов органических соединений является актуальной проблемой.

Цель работы - разработка простых и доступных методов синтеза новых [хиназолин-4(3Н)-илиден]гидразидов циклоалкил-(гетарил-)карбоновых кислот, изучение их физико-химических свойств, исследование противомикробной и противогрибковой активности.

Материалы и методы. Изучение противомикробной активности синтезированных соединений проводили методом двукратных серийных разведений в бульоне Мюллера-Хинтона (для штаммов Staphylococcus aureus ATCC 25923, Escherichia coli ATCC 25922, Pseudomonas aeruginosa ATCC 27853) и в бульоне Сабуро (для Candida albicans ATCC 885-653). Определяли МИК (минимальная ингибирующая концентрация), МБцК и МФцК (минимальная бактерицидная и фунгицидная концентрации соответственно).

Результаты. Установлено, что синтезированные соединения проявляют противомикробную и противогрибковую активность к исследуемым штаммам.

Выводы. Рассмотрены препаративные методы синтеза [хиназолин-4(3Н)-илиден]гидразидов циклоалкил-(гетарил-) карбоновых кислот, которые представляют ценность как химические реагенты для получения конденсированных гетероциклических и биологически активных соединений. Строение и индивидуальность подтверждены с помощью элементного анализа и физико-химических методов ( ${ }^{1} \mathrm{H}$ ЯМР-спектроскопия, хромато-масс-спектрометрия). Анализ результатов микробиологического исследования показывает, что среди синтезированных соединений наибольшую противомикробную активность проявляют $N^{\prime}$-[хиназолин-4(3Н)-илиден]-2(3)-гетерил-гидразиды, содержащие в молекуле остаток пиридинкарбоновой кислоты, что превышает активность эталона сравнения триметоприма. Наибольшую противогрибковую активность проявляет $N^{\prime}$-(6-бромохиназолин-4(3Н)-илиден)бензофурил-2-карбогидразид, который сопоставим с эталоном сравнения кетоконазолом.

The modern standards and algorithms of antimicrobial therapy, that ensure a high level of the treatment's quality and prevention of the most infectious-inflammatory diseases, provide the wide using of chemotherapeutic agents.

However, the modern medicines do not always have satisfactory chemotherapeutic and pharmacological properties; this fact is most often associated with the resistance of microorganisms to them.

That is why the creation of the new chemotherapeutic drugs by natural antibiotics' chemical and microbiological modification, the chemical synthesis of the new substances among the various classes of organic compounds is a topical problem [1].

The quinazoline derivatives belong to one of the most perspective types of heterocyclic compounds that are used in modern pharmaceutical practice.

Sufficiently ample opportunity of the quinazoline cycle's chemical modification creates the significant prerequisites for the design of the new potential medical drugs [2]. The results of the previous studies [3], showed that some (3H-quinazolin-4-ylidene)hydrazides of carboxylic acids have the expressed antimicrobial, antifungal and other activities.

This fact substantiates the reasonability of the further researches aimed at the creation of new antimicrobial medical drugs with the greater efficiency and less toxicity in comparison with the available on the pharmaceutical market drugs.

Moreover, hydrazides of carboxylic acids are an interesting group of the organic compounds [4]. This group due to the combination of two reaction centers in the molecule is widely used in cyclocondensation reactions for the heterocyclic systems' forming [5].

\section{The aim}

The aim of the study is the developing of the simple and accessible methods for the synthesis of new [quinazoline4(3H)-ylidene]hydrazides of cycloalkyl-(hetaryl) carboxylic acids, analysis of their physical-chemical properties, antimicrobial and antifungal activity.

\section{Materials and methods}

The experimental chemical part. Melting points were determined in open capillary tubes and were uncorrected. The elemental analyses $(\mathrm{C}, \mathrm{H}, \mathrm{N}, \mathrm{S})$ were performed using the ELEMENTAR vario EL Cube analyzer (USA). Analyses were indicated by the symbols of the elements or functions within $\pm 0.3 \%$ of the theoretical values. The ${ }^{1} \mathrm{H}$ NMR spectra $(400 \mathrm{MHz})$ and ${ }^{13} \mathrm{C}$ NMR spectra $(100 \mathrm{MHz})$ were recorded on a Varian-Mercury 400 (Varian Inc., Palo Alto, CA, USA) spectrometer with TMS as the internal standard in DMSO-d6 solution. The LC-MS were recorded using a chromato-mass spectrometric system which consisted of a high-performance liquid chromatograph "Agilent 1100 Series" (Agilent, Palo Alto, CA, USA) equipped with a diode-matrix and mass-selective detector "Agilent LC/MSD $S L "$ (atmospheric pressure chemical ionization - APCI). The purity of all obtained compounds was checked by ${ }^{1} \mathrm{H}-\mathrm{NMR}$ and LC-MS.

Synthesis of the derivatives of 4-hydrazinoquinazoline 
(1.1-1.5) and 4-chloroquinazoline (2.1-2.5) was implemented by well-known methods, with constants that correspond to literature data [6].

Methods of synthesis of N'-(quinazolin-4(3H)-ylidene) carbohydrazides (3.1-3.25).

Method A. $1.95 \mathrm{~g}(11 \mathrm{mmol})$ of carbonyldiimidazole was added to the solution of $11 \mathrm{mmol}$ of the corresponding carboxylic acid in $10 \mathrm{ml}$ of anhydrous dioxane. The formed mixture was heated on a water bath at $60-80^{\circ} \mathrm{C}$ for 1 hour, protecting from the air moisture, using tube filled by calcium chloride. Then $10 \mathrm{mmol}$ of the corresponding substituted 4-hydrazinoquinazolines (1.1-1.5) were added to the reaction mixture with stirring. Then it was left for 8 hours at ambient temperature. The formed mixture was poured into water and acidified by acetic acid to $\mathrm{pH} \mathrm{6-7.} \mathrm{The} \mathrm{formed}$ precipitate was filtered off, dried, crystallized if necessary.

Method B. $11 \mathrm{mmol}$ of the corresponding carboxylic acid hydrazide was added to the solution of $10 \mathrm{mmol}$ of the corresponding substituted 4-chloroquinazoline (2.1-2.5) in $10 \mathrm{ml}$ of dioxane; kept it on a water bath at $60^{\circ} \mathrm{C}$ for 8 hours. After cooling, the reaction mixture was poured into water, alkalified to $\mathrm{pH} 6-7$ by $5 \%$ sodium hydrocarbonate solute. The formed precipitate was filtered off, dried, crystallized if necessary.

N'-(7-fluoroquinazolin-4(3H)-ylidene)cyclopropanecarbohydrazide (3.1). Yield: $86.90 \%$ (Method A), mp 122-124 ${ }^{\circ} \mathrm{C}$; ' ${ }^{1} \mathrm{H}$ NMR: $\delta=0.95-0.65$ (m, 4H, cyclopropyl H-2,2', H-3,3'), 1.72-1.83 (m, 1H, cyclopropyl H-1), 6.85 (d, J=7.6 $\mathrm{Hz}, 1 \mathrm{H}, \mathrm{H}-8), 6.91$ (d, J = $8.8 \mathrm{~Hz}, 1 \mathrm{H}, \mathrm{H}-6), 7.97-7.84$ (m, 1H, H-5), 8.43 (s, 1H, H-2), 10.14 (s, 1H, NH), 11.40 (s, $1 \mathrm{H}, \mathrm{NH})$; LC-MS, m/z = 247 [M+1]; Anal. Calcd for $\mathrm{C}_{12} \mathrm{H}_{11} \mathrm{FN}_{4} \mathrm{O}$ : C, 58.53; H, 4.50; N, 22.75; Found: C, 58.54; $\mathrm{H}, 4.49 ; \mathrm{N}, 22.77$.

N'-(6-chloroquinazolin-4(3H)-ylidene)cyclopropanecarbohydrazide (3.2). Yield: $92.12 \%$ (Method A), mp 130-132 ${ }^{\circ} \mathrm{C}$; ' $\mathrm{H}$ NMR: $\delta=0.97-0.63$ (m, 4H, cyclopropyl H-2,2', H-3,3'), 1.67-1.84 (m, 1H, cyclopropyl H-1), 7.11 (d, J = 7.6 $\mathrm{Hz}, 1 \mathrm{H}, \mathrm{H}-8$ ), 7.33 (d, J = 8.2 Hz, 1H, H-5), 7.76 (d, J= 8.0 $\mathrm{Hz}, 1 \mathrm{H}, \mathrm{H}-7$ ), 8.46 (d, J = $7.7 \mathrm{~Hz}, 1 \mathrm{H}, \mathrm{H}-2$ ), 10.15 (s, 1H, $\mathrm{NH}), 11.46$ (s, 1H, NH); LC-MS, m/z = 263 [M+1]; Anal. Calcd for $\mathrm{C}_{12} \mathrm{H}_{11} \mathrm{CIN}_{4} \mathrm{O}$ : C, 54.87; H, 4.22; N, 21.33; Found: C, 54.85; H, 4.23; N, 21.31.

N'-(quinazolin-4(3H)-ylidene)adamantane-1-carbohydrazide (3.3). Yield: $99.1 \%$ (Method A), mp 221-223 ${ }^{\circ} \mathrm{C}$; ${ }^{1} \mathrm{H}$ NMR: $\delta=1.75(\mathrm{~d}, \mathrm{~J}=3.6 \mathrm{~Hz}, 6 \mathrm{H}$, adamantyl-1 H-4,4', H-6,6', H-10,10'), 1.92 (s, 6H, adamantyl-1 H-2,2', H-8,8', H-9,9'), 2.06 (s, 3H, adamantyl-1 H-3, H-5, H-7), 7.51 -6.88 (m, 3H, H-6, H-7, H-8), 7.72 (s, 1H, H-5), 7.93 (s, 1H, H-2), 10.01 (s, 1H, NH), 11.44 (s, 1H, NH); LC-MS, m/z = 323 $[\mathrm{M}+1]$; Anal. Calcd for $\mathrm{C}_{19} \mathrm{H}_{22} \mathrm{~N}_{4} \mathrm{O}: \mathrm{C}, 70.78 ; \mathrm{H}, 6.88 ; \mathrm{N}$, 17.38; Found: C, 70.79; H, 6.86; N, 17.38 .

N'-(8-methylquinazolin-4(3H)-ylidene)adamantane-1-carbohydrazide (3.4). Yield: $90.95 \%$ (Method A), mp 256-258 ${ }^{\circ} \mathrm{C}$; ${ }^{1} \mathrm{H}$ NMR: $\delta=1.75$ (s, 9H, adamantyl-1 $\mathrm{H}-4,4$ ', H-6,6', H-10,10', - $\mathrm{CH}_{3}$ ), 1.94 (s, 6H, adamantyl-1 $\mathrm{H}-2,2^{\prime}, \mathrm{H}-8,8$ ', H-9,9'), 2.17-2.02 (m, 3H, adamantyl-1 H-3, $\mathrm{H}-5, \mathrm{H}-7$ ), $8.63-6.76$ (m, 4H, H-2, H-5, H-6, H-7), 9.90 (s, $1 \mathrm{H}, \mathrm{NH}), 10.87$ (s, 1H, NH); LC-MS, m/z = 337 [M+1]; Anal. Calcd for $\mathrm{C}_{20} \mathrm{H}_{24} \mathrm{~N}_{4} \mathrm{O}: \mathrm{C}, 71.40 ; \mathrm{H}, 7.19 ; \mathrm{N}, 16.65$; Found: C, $71.39 ; \mathrm{H}, 7.20 ; \mathrm{N}, 16.67$.

N'-(7-fluoroquinazolin-4(3H)-ylidene)adamantane-1-carbohydrazide (3.5). Yield: $98.9 \%$ (Method A), mp
225-227 ${ }^{\circ} \mathrm{C} ;{ }^{1} \mathrm{H}$ NMR: $\delta=2.22-1.55$ (m, $15 \mathrm{H}$, adamantyl-1 H-2,2', H-3, H-4,4', H-5, H-6,6', H-7,H-8,8', H-9,9', H-10,10'), $6.89(\mathrm{~d}, J=8.8 \mathrm{~Hz}, 1 \mathrm{H}, \mathrm{H}-8), 7.30(\mathrm{~d}, J=7.8 \mathrm{~Hz}, 1 \mathrm{H}, \mathrm{H}-6)$, $8.08-7.65(\mathrm{~m}, 1 \mathrm{H}, \mathrm{H}-5), 8.41$ (s, 1H, H-2), $9.98(\mathrm{~s}, 1 \mathrm{H}, \mathrm{NH})$, 11.48 (s, 1H, NH); LC-MS, m/z = 341 [M+1]; Anal. Calcd for $\mathrm{C}_{19} \mathrm{H}_{21} \mathrm{FN}_{4} \mathrm{O}$ : C, 67.04; $\mathrm{H}, 6.22 ; \mathrm{N}, 16.46$; Found: C, 67.03; $\mathrm{H}, 6.20 ; \mathrm{N}, 16.45$.

N'-(6-chloroquinazolin-4(3H)-ylidene)adamantane-1-carbohydrazide (3.6). Yield: $98.4 \%$ (Method A), mp 239-241 ${ }^{\circ} \mathrm{C}$; ${ }^{1} \mathrm{H}$ NMR: $\delta=2.16-1.58$ (m, $15 \mathrm{H}$, adamantyl-1 $\mathrm{H}-2,2^{\prime}, \mathrm{H}-3, \mathrm{H}-4,4$ ', $\mathrm{H}-5, \mathrm{H}-6,6^{\prime}, \mathrm{H}-7, \mathrm{H}-8,8^{\prime}, \mathrm{H}-9,9^{\prime}, \mathrm{H}-10,10^{\prime}$ ), 7.13 (s, 1H, H-8), 7.95-7.58 (m, 2H, H-5, H-7), 8.44 (s, 1H, $\mathrm{H}-2$ ), 9.98 (s, 1H, NH), 11.55 (s, 1H, NH); LC-MS, m/z = 357 $[\mathrm{M}+1]$; Anal. Calcd for $\mathrm{C}_{19} \mathrm{H}_{21} \mathrm{CIN}_{4} \mathrm{O}$ : C, 63.95; $\mathrm{H}, 5.93$; N, 15.70; Found: C, 63.96; H, 5.95; N, 15.69.

N'-(8-methylquinazolin-4(3H)-ylidene)furan-3-carbohydrazide (3.7). Yield: $95.42 \%$ (Method A), mp 228-230 ${ }^{\circ} \mathrm{C}$; ${ }^{1} \mathrm{H}$ NMR: $\delta=2.53\left(\mathrm{~s}, 3 \mathrm{H},-\mathrm{CH}_{3}\right), 6.92(\mathrm{~s}, 1 \mathrm{H}$, furan-3-yl $\mathrm{H}-4), 7.77$ - 7.10 (m, 3H, H-6,7, furan-3-yl H-5), 8.54-8.10 (m, 3H, H-2, H-5, furan-3-yl H-2), 10.24 (s, 1H, NH), 10.94 (s, 1H, NH); LC-MS, m/z = 269 [M+1]; Anal. Calcd for $\mathrm{C}_{14} \mathrm{H}_{12} \mathrm{~N}_{4} \mathrm{O}_{2}$ : C, 62.68; $\mathrm{H}, 4.51$; N, 20.88; Found: C, 62.67; $\mathrm{H}, 4.53 ; \mathrm{N}, 20.86$.

N'-(7-fluoroquinazolin-4(3H)-ylidene)furan-3-carbohydrazide (3.8). Yield: $90.36 \%$ (Method A), mp 236-238 ${ }^{\circ} \mathrm{C}$; ${ }^{1} \mathrm{H}$ NMR: $\delta=7.09-6.77$ (m, 2H, H-6, furan-3-yl H-4), 7.29 (s, 1H, H-8), 7.57 (d, J=8.0 Hz, 1H, furan-3-yl H-5), 7.87 (d, $J=8.1 \mathrm{~Hz}, 1 \mathrm{H}, \mathrm{H}-5), 8.65-8.07$ (m, 2H, H-2, furan-3-yl H-2), 10.28 (s, 1H, NH), 11.56 (s, 1H, NH); LC-MS, m/z = 273 $[\mathrm{M}+1]$; Anal. Calcd for $\mathrm{C}_{13} \mathrm{H}_{9} \mathrm{FN}_{4} \mathrm{O}_{2}: \mathrm{C}, 57.36 ; \mathrm{H}, 3.33 ; \mathrm{N}$, 20.58; Found: C, 57.38; H, 3.32; N, 20.57.

N'-(6-chloroquinazolin-4(3H)-ylidene)furan-3-carbohydrazide (3.9). Yield: $92.14 \%$ (Method A), mp $258-260{ }^{\circ} \mathrm{C} ;{ }^{1} \mathrm{H}$ NMR: $\delta=6.92(\mathrm{~s}, 1 \mathrm{H}$, furan-3-yl H-4), $7.16(\mathrm{~d}, J=8.8 \mathrm{~Hz}$, $1 \mathrm{H}, \mathrm{H}-8), 7.37$ (d, J = 9.2 Hz, $1 \mathrm{H}, \mathrm{H}-7), 7.99-7.51(\mathrm{~m}, 2 \mathrm{H}$, $\mathrm{H}-5$, furan-3-yl H-5), 8.23 (s, 1H, H-2), 8.49 (s, 1 H, furan-3-yl $\mathrm{H}-2), 10.29$ (s, 1H, NH), $11.83-11.49$ (m, 1H, NH); LC-MS, $\mathrm{m} / \mathrm{z}=289[\mathrm{M}+1]$; Anal. Calcd for $\mathrm{C}_{13} \mathrm{H}_{9} \mathrm{CIN}_{4} \mathrm{O}_{2}: \mathrm{C}, 54.09 ; \mathrm{H}$, 3.14; N, 19.41; Found: C, 54.07; H, 3.15; N, 19.41 .

N'-(6-bromoquinazolin-4(3H)-ylidene)furan-3-carbohydrazide (3.10). Yield: $76.54 \%$ (Method A), mp 237-239 ${ }^{\circ} \mathrm{C}$; ${ }^{1} \mathrm{H}$ NMR: $\delta=6.91$ (s, 1H, furan-3-yl H-4), $7.74-7.44$ (m, $2 \mathrm{H}, \mathrm{H}-7, \mathrm{H}-8), 7.93-7.75$ (m, 1H, furan-3-yl H-5), 7.97 (d, $J=3.2 \mathrm{~Hz}, 1 \mathrm{H}, \mathrm{H}-5), 8.22(\mathrm{~s}, 1 \mathrm{H}, \mathrm{H}-2), 8.47$ (s, $1 \mathrm{H}$ furan3-yl H-2), 10.27 (d, J = 8.4 Hz, 1H, NH), 11.62 (s, 1H, NH); LC-MS, $\mathrm{m} / \mathrm{z}=289$ [M+1]; Anal. Calcd for $\mathrm{C}_{13} \mathrm{H}_{9} \mathrm{BrN}_{4} \mathrm{O}_{2}: \mathrm{C}$, 46.87; H, 2.72; N, 16.82; Found: C, 46.85; H, 2.71; N, 16.83 .

N'-(quinazolin-4(3H)-ylidene)thiophene-2-carbohydrazide (3.11). Yield: $99.27 \%$ (Method A), 52.7 (Method B), mp 205-207 ${ }^{\circ} \mathrm{C}$; ${ }^{1} \mathrm{H}$ NMR: $\delta=8.46-7.07$ (m, 8H, H-2, H-5, H-6, $\mathrm{H}-7, \mathrm{H}-8$, thiophene-2-yl H-3, H-4, H-5), $10.67 / 9.94$ (b.s, $1 \mathrm{H}, \mathrm{NH}), 11.76$ (s, 1H, NH); LC-MS, m/z = 271 [M+1], 273 [M+3]; Anal. Calcd for $\mathrm{C}_{13} \mathrm{H}_{10} \mathrm{~N}_{4} \mathrm{OS}$ : C, 57.76; H, 3.73; N, 20.73; S, 11.86; Found: C, 57.76; H, 3.71; N, 20.74; S, 11.88. N'-(7-fluoroquinazolin-4(3H)-ylidene)thiophene-2-carbohydrazide (3.12). Yield: $93.65 \%$ (Method A), mp 259$261^{\circ} \mathrm{C} ;{ }^{1} \mathrm{H}$ NMR: $\delta=6.91(\mathrm{~d}, J=8.6 \mathrm{~Hz}, 1 \mathrm{H}, \mathrm{H}-8), 7.04(\mathrm{~d}$, $J=8.4 \mathrm{~Hz}, 1 \mathrm{H}, \mathrm{H}-6), 7.33-7.09$ (m, 1H, thiophene-2-yl $\mathrm{H}-4), 7.94-7.57$ (m, 2H, thiophene-2-yl H-3, H-5), 8.06 (d, $J=8.4 \mathrm{~Hz}, 1 \mathrm{H}, \mathrm{H}-5$ ), 8.29 (s, $1 \mathrm{H}, \mathrm{H}-2$ ), 10.53/9.80 (s, $1 \mathrm{H}, \mathrm{NH}), 11.62$ (s, 1H, NH); LC-MS, m/z = 289 [M+1]; Anal. Calcd for $\mathrm{C}_{13} \mathrm{H}_{9} \mathrm{FN}_{4} \mathrm{OS}$ : C, 54.16; H, 3.15; F, 6.59; N, 19.43; 
O, 5.55; S, 11.12; Found: C, 54.18; H, 3.14; F, 6.58; N, $19.45 ;$ O, 5.54; S, 11.11 .

N'-(8-methylquinazolin-4(3H)-ylidene)thiophene-3-carbohydrazide (3.13). Yield: $73.85 \%$ (Method A), mp 228-230 ${ }^{\circ} \mathrm{C}$; ${ }^{1} \mathrm{H}$ NMR: $\delta=2.49\left(\mathrm{~s}, 3 \mathrm{H}, \mathrm{CH}_{3}\right), 7.82-7.11(\mathrm{~m}, 4 \mathrm{H}, \mathrm{H} 6$, $\mathrm{H} 7$, thiophene-3-yl H-4, H-5), $8.56-7.96$ (m, 3H, H-2, H-5, thiophene-3-yl H-2), 10.37 (s, 1H, NH); LC-MS, m/z = 285 $[\mathrm{M}+1]$; Anal. Calcd for $\mathrm{C}_{14} \mathrm{H}_{12} \mathrm{~N}_{4} \mathrm{OS}$ : C, 59.14; $\mathrm{H}, 4.25 ; \mathrm{N}$, 19.70; S, 11.28; Found: C, 59.12; H, 4.26; N, 19.71; S, 11.27. N'-(7-fluoroquinazolin-4(3H)-ylidene)thiophene-3-carbohydrazide (3.14). Yield: $93.65 \%$ (Method A), mp 228-230 ${ }^{\circ} \mathrm{C}$; ${ }^{1} \mathrm{H}$ NMR: $\delta=8.66-6.79$ (m, 7H, H-2, H-5, H-6, H-8, thiophene-3-yl H-2, H-4, H-5), 10.33 (s, 1H, NH), 11.58 (s, $1 \mathrm{H}, \mathrm{NH})$; LC-MS, m/z = $285[\mathrm{M}+1]$; Anal. Calcd for $\mathrm{C}_{13} \mathrm{H}-$ ${ }_{9} F_{4}$ OS: C, 54.16; H, 3.15; N, 19.43; S, 11.12; Found: C, 54.15; H, 3.17; N, 19.41; S, 11.13.

N'-(6-chloroquinazolin-4(3H)-ylidene)thiophene-3-carbohydrazide (3.15). Yield: $86.00 \%$ (Method A), mp 262-264 ${ }^{\circ} \mathrm{C}$; ${ }^{1} \mathrm{H}$ NMR: $\delta=8.70-7.05$ (m, 7H, H-2, H-5, H-7, H-8, thiophene-3-yl H-2, H-4, H-5), 10.41 (s, 1H, NH), 11.62 (s, $1 \mathrm{H}, \mathrm{NH})$; LC-MS, m/z = 305 [M+1]; Anal. Calcd for $\mathrm{C}_{13} \mathrm{H}-$ ${ }_{9} \mathrm{CIN}_{4} \mathrm{OS}$ : C, 51.24; H, 2.98; N, 18.38; S, 10.52; Found: C, 51.24; H, 2.99; N, 18.36; S, 10.53.

N'-(6-bromoquinazolin-4(3H)-ylidene)thiophene-3-carbohydrazide (3.16). Yield: $65.86 \%$ (Method A), mp 249-251 ${ }^{\circ} \mathrm{C}$; ${ }^{1} \mathrm{H}$ NMR: $\delta=8.75-7.35(\mathrm{~m}, 7 \mathrm{H}, \mathrm{H}-2, \mathrm{H}-5, \mathrm{H}-7, \mathrm{H}-8$, thiophene-3-yl H-2, H-4, H-5), 10.42 (s, 1H, NH), 11.63 (s, $1 \mathrm{H}, \mathrm{NH}) ; \mathrm{LC}-\mathrm{MS}, \mathrm{m} / \mathrm{z}=350$ [M+1]; Anal. Calcd for $\mathrm{C}_{13} \mathrm{H}_{9} \mathrm{Br}-$ $\mathrm{N}_{4} \mathrm{OS}$ : C, 44.71; H, 2.60; N, 16.04; S, 9.18; Found C, 44.69; $\mathrm{H}, 2.61 ; \mathrm{N}, 16.03 ; \mathrm{S}, 9.20$.

N'-(quinazolin-4(3H)-ylidene)benzofuran-2-carbohydrazide (3.17). Yield: $92.56 \%$ (Method A), 62.5 (Method B), mp 286-288 ${ }^{\circ} \mathrm{C}$; ${ }^{1} \mathrm{H}$ NMR: $\delta=7.25(\mathrm{~d}, 1 \mathrm{H}, \mathrm{J}=8.4 \mathrm{~Hz}$, benzofuran-2-yl H-4), 7.37 (m, 2H, benzofuran-2-yl H-5, $\mathrm{H}-6), 7.50$ (t, $1 \mathrm{H}, \mathrm{J}=7.8 \mathrm{~Hz}, \mathrm{H}-7), 7.57(\mathrm{~T}, 1 \mathrm{H}, \mathrm{J}=7.8 \mathrm{~Hz}$, $\mathrm{H}-6), 7.64(\mathrm{~s}, 1 \mathrm{H}$, benzofuran-2-yl H-3), $7.75(\mathrm{~d}, 1 \mathrm{H}, \mathrm{J}=8.4$ $\mathrm{Hz}$, benzofuran-2-yl H-7申), $7.80(\mathrm{~d}, 1 \mathrm{H}, \mathrm{J}=7.8 \mathrm{~Hz}, \mathrm{H}-8)$, 8.00 (d, 1H, J = 7.9 Hz, H-5), 8.12 (s, 1H, H-2), 11.03 (s, $1 \mathrm{H}, \mathrm{NH}), 11.96$ (s, 1H, NH); LC-MS, m/z = 305 [M+1]; Anal. Calcd for $\mathrm{C}_{17} \mathrm{H}_{12} \mathrm{~N}_{4} \mathrm{O}_{2}: \mathrm{C}, 67.10 ; \mathrm{H}, 3.97 ; \mathrm{N}, 18.41$; Found: C, $67.08 ; \mathrm{H}, 3.98 ; \mathrm{N}, 18.39$.

N'-(8-methylquinazolin-4(3H)-ylidene)benzofuran-2-carbohydrazide (3.18). Yield: $84.81 \%$ (Method $\mathrm{A}$ ), mp 284-286 ${ }^{\circ} \mathrm{C}$; ${ }^{1} \mathrm{H}$ NMR: $\delta=2.50\left(\mathrm{~s}, 3 \mathrm{H}, \mathrm{CH}_{3}\right), 8.03-7.12$ (m, 9H, H-2, H-5, H-6, H-7, benzofuran-2-yl H-3, H-4, H-5, $\mathrm{H}-6, \mathrm{H}-7), 11.05$ (s, 1H, NH); LC-MS, m/z = 319 [M+1]; Anal. Calcd for $\mathrm{C}_{18} \mathrm{H}_{14} \mathrm{~N}_{4} \mathrm{O}_{2}$ : C, 67.92; $\mathrm{H}, 4.43 ; \mathrm{N}, 17.60$; Found: C, $67.90 ; \mathrm{H}, 4.43 ; \mathrm{N}, 17.59$.

N'-(7-fluoroquinazolin-4(3H)-ylidene)benzofuran-2-carbohydrazide (3.19). Yield: $92.00 \%$ (Method A), mp 309-311 ${ }^{\circ} \mathrm{C}$; ${ }^{1} \mathrm{H}$ NMR: $\delta=7.81-6.72(\mathrm{~m}, 9 \mathrm{H}, \mathrm{H}-2, \mathrm{H}-5, \mathrm{H}-6, \mathrm{H}-8$, benzofuran-2-yl H-3, H-4, H-5, H-6, H-7), $11.70(\mathrm{~s}, 1 \mathrm{H}, \mathrm{NH})$; LC-MS, m/z = $323[\mathrm{M}+1]$; Anal. Calcd for $\mathrm{C}_{17} \mathrm{H}_{11} \mathrm{FN}_{4} \mathrm{O}_{2}: \mathrm{C}$, 63.35; H, 3.44; N, 17.38; Found: C, 63.34; H, 3.46; N, 17.37 .

N'-(6-chloroquinazolin-4(3H)-ylidene)benzofuran-2-carbohydrazide (3.20). Yield: $85.00 \%$ (Method A), mp 294-296 ${ }^{\circ} \mathrm{C}$; ${ }^{1} \mathrm{H}$ NMR: $\delta=7.78-7.15(\mathrm{~m}, 7 \mathrm{H}, \mathrm{H}-5, \mathrm{H}-8$, benzofuran-2-yl H-3, H-4, H-5, H-6, H-7), $7.98(\mathrm{~d}, \mathrm{~J}=8.0 \mathrm{~Hz}, 1 \mathrm{H}$, $\mathrm{H}-7), 8.43$ (s, 1H, H-2), 10.90 (s, 1H, NH), 11.80 (s, 1H, NH); LC-MS, $\mathrm{m} / \mathrm{z}=339$ [M+1]; Anal. Calcd for $\mathrm{C}_{17} \mathrm{H}_{11} \mathrm{CIN}_{4} \mathrm{O}_{2}: \mathrm{C}$, 60.28; H, 3.27; N, 16.54; Found: C, 60.27; H, 3.26; N, 16.55 . N'-(6-bromoquinazolin-4(3H)-ylidene)benzofu- ran-2-carbohydrazide (3.21). Yield: $68.50 \%$ (Method A), mp 267-269 ${ }^{\circ} \mathrm{C}$; ${ }^{1} \mathrm{H}$ NMR: $\delta=8.43-7.05$ (m, 9H, H-2, H-5, $\mathrm{H}-7, \mathrm{H}-8$, benzofuran-2-yl H-3, H-4, H-5, H-6, H-7), 10.90 (s, 1H, NH), 11.79 (s, 1H, NH); LC-MS, m/z = 384 [M+1]; Anal. Calcd for $\mathrm{C}_{17} \mathrm{H}_{11} \mathrm{BrN}_{4} \mathrm{O}_{2}: \mathrm{C}, 53.28 ; \mathrm{H}, 2.89 ; \mathrm{N}, 14.62$; Found: C, 53.27; H, 2.90; N, 14.64.

N'-(quinazolin-4(3H)-ylidene)picolinohydrazide (3.22). Yield: $94.87 \%$ (Method A), mp 270-272 ${ }^{\circ} \mathrm{C}$; ${ }^{1} \mathrm{H}$ NMR: $\delta=7.17(\mathrm{~d}, J=7.4 \mathrm{~Hz}, 1 \mathrm{H}, \mathrm{H}-8), 7.36(\mathrm{t}, J=7.9 \mathrm{~Hz}, 1 \mathrm{H}$, $\mathrm{H}-6), 7.50(\mathrm{t}, J=6.0 \mathrm{~Hz}, 1 \mathrm{H}, \mathrm{H}-7), 7.59(\mathrm{t}, J=8.2 \mathrm{~Hz}, 1 \mathrm{H}$, picolinoyl H-5), 7.99 (d, J = 8.4 Hz, 1H, H-5), $8.25(\mathrm{~m}, 1 \mathrm{H}$, picolinoyl H-3,4), 8.61 (s, 1H, H-2), $8.73(\mathrm{~d}, J=6.6 \mathrm{~Hz}, 1 \mathrm{H}$, picolinoyl H-6), 11.46 (s, 1H, NH); LC-MS, m/z = 266 [M+1]; Anal. Calcd for $\mathrm{C}_{14} \mathrm{H}_{11} \mathrm{~N}_{5} \mathrm{O}$ : C, 63.39; H, 4.18; N, 26.40; Found: C, 63.41; H, 4.19; N, 26.42.

N'-(quinazolin-4(3H)-ylidene)nicotinohydrazide (3.23). Yield: $98.00 \%$ (Method A), mp 251-253 ${ }^{\circ} \mathrm{C}$; ${ }^{1} \mathrm{H}$ NMR: $\delta=8.44-7.06(\mathrm{~m}, 7 \mathrm{H}, \mathrm{H}-5, \mathrm{H}-6, \mathrm{H}-7, \mathrm{H}-8$, nicotinoyl $\mathrm{H}-3$, $\mathrm{H}-4, \mathrm{H}-5), 8.69$ (s, 1H, H-2), 9.09 (s, 1H, nicotinoyl H-2), 10.72 (s, 1H, NH); LC-MS, m/z = 266 [M+1]; Anal. Calcd for $\mathrm{C}_{14} \mathrm{H}_{11} \mathrm{~N}_{5} \mathrm{O}$ : C, 63.39; $\mathrm{H}, 4.18$; N, 26.40; Found: C, 63.37; H, 4.19; N, 26.41.

N'-(quinazolin-4(3H)-ylidene)isonicotinohydrazide (3.24). Yield: $83.30 \%$ (Method A), mp 255-257 ${ }^{\circ} \mathrm{C} ;{ }^{1} \mathrm{H}$ NMR: $\delta=8.84-7.05(\mathrm{~m}, 9 \mathrm{H}, \mathrm{H}-2, \mathrm{H}-5, \mathrm{H}-6, \mathrm{H}-7, \mathrm{H}-8$, isonicotinoyl $\mathrm{H}-2, \mathrm{H}-3, \mathrm{H}-5, \mathrm{H}-6), 10.76$ (s, 1H, NH), 11.62 (s, 1H, NH); LC-MS, m/z = $266[\mathrm{M}+1]$; Anal. Calcd for $\mathrm{C}_{14} \mathrm{H}_{11} \mathrm{~N}_{5} \mathrm{O}: \mathrm{C}$, 63.39; H, 4.18; N, 26.40; Found: C, 63.36; H, 4.20; N, 26.41.

$N^{\prime}$-(6-bromoquinazolin-4(3H)-ylidene)isonicotinohydrazide (3.25). Yield: $61.01 \%$ (Method A), mp $245-247^{\circ} \mathrm{C} ;{ }^{1} \mathrm{H}$ NMR: $\delta=8.24-7.49(\mathrm{~m}, 6 \mathrm{H}, \mathrm{H}-5, \mathrm{H}-6, \mathrm{H}-7, \mathrm{H}-8$, isonicotinoyl H-3, H-5), $8.49(\mathrm{~s}, 1 \mathrm{H}, \mathrm{H}-2), 8.71(\mathrm{~d}, \mathrm{~J}=8.6 \mathrm{~Hz}, 2 \mathrm{H}$, isonicotinoyl H-2, H-6), $10.79(\mathrm{~s}, 1 \mathrm{H}, \mathrm{NH}), 11.79(\mathrm{~s}, 1 \mathrm{H}, \mathrm{NH})$; LC-MS, m/z = 344 [M+1]; Anal. Calcd for $\mathrm{C}_{14} \mathrm{H}_{10} \mathrm{BrN}_{5} \mathrm{O}: \mathrm{C}$, 48.86; H, 2.93; N, 20.35; Found: C, 48.85; H, 2.92; N, 20.36 .

Synthesized compounds (3.1-3.25) are white (3.1,3.2), yellow (3.3-3.25) crystalline substances, insoluble in water, slightly soluble in alcohols, soluble in dioxane and DMF. The compounds 3.1-3.9, were purified by recrystallization from the mixture of dioxane-water (1:1), DMF-water (1:1) for the analysis.

The experimental biological part. The sensitivity of the microorganisms to the synthesized compounds was evaluated according the described methods [7]. The assay was conducted on Mueller-Hinton medium by two-fold serial dilution of the compound in $1 \mathrm{ml}$, after that $0.1 \mathrm{ml}$ of microbial seeding (106 cells $/ \mathrm{ml}$ ) was added. The minimal inhibitory concentration of the compound was determined by the absence of visual growth in the test tube with a minimal concentration of the substance, and then the minimal bactericide/fungicide concentration was determined by the absence of growth on agar after inoculation of the microorganism from the transparent test tubes. Dimethylsulfoxide was used as a solvent, with an initial solution concentration of $1 \mathrm{mg} / \mathrm{ml}$. Preliminary screening was performed on Staphylococcus aureus ATCC 25923, Escherichia coli ATCC 25922, Pseudomonas aeruginosa ATCC 27853, and Candida albicans ATCC 885-653 standard test cultures. Nitrofual, Trimetoprim and Ketoconazole were used as the reference compounds with proven antibacterial/antifungal activity. Additional quality control of the culture medium and solvents was conducted by commonly used methods $[8,9]$. 


\section{Results and its discussion}

Synthesis of (3H-quinazolin-4-ylidene)hydrazides cycloalkyl-(hetaryl) carboxylic acid 3.1-3.25 as the convenient precursors for the preparation of the corresponding tricyclic derivatives [10], was carried out in two alternative ways: by acylation of the corresponding substituted 4-hydrazinoquinazolines (1.1-1.5) by activated cycloalkyl-(hetaryl) carboxylic acids (Method A, scheme 1) and by interaction substituted 4-chloroquinazolines (2.1-2.5) with the hydrazides of the corresponding acids (Method B, scheme 1) $[11,12]$. The acylation of the compounds $1.1-1.5$ was realized by imidazolides of alicyclic or heterocyclic acids (Method A). It should be noted that the regioselectivity of the reaction is determined by the place of the primary nucleophilic attack, that may be represented by the final nitrogen atom of the hydrazine moiety or by the endocyclic N (1) and $\mathrm{N}$ (3) atoms of quinazoline. Later, the alternative synthesis of some $\mathrm{N}$-acyl derivatives $(3.11,3.17)$ was carried out, it was based on the interaction of substituted 4-chloroquinazolines (2.1-2.5) with hydrazides of the corresponding alicyclic acids (Method B). The using of this approach allowed the definitely establishing of the reactions 'direction.

Each of the mentioned approaches has both advantages and disadvantages. Among the characteristics of the carbonyldiimidazole method, it is necessary to note the rate of performing, high yields and purity of the final products, which, in our opinion, are significant advantages, despite of the necessity of anhydrous solvents' using. One of the features of this method is the possible one-stage heterocyclization of the corresponding hydrazides into triazoloquinazoline systems, provided by the extended time and the increased temperature regime of the reaction. But the chosen reaction conditions made it possible to get the individual N'-[quinazoline-4(3H)-ylidene]-2-(3)-cycloalkyl(hetaryl)-hydrazides with the high yields.

Among the disadvantage of the second method, it is necessary to note the duration of hydrazides of alicyclic or heterocyclic acids' synthesis and also the using of shortlived substituted 4-chloroquinazolines (2.1-2.5) as initial compounds.

In the LC-MS spectra (APCI) of the compounds $3.1-3.25$, the signals of positive ions $[M+1]$ were recorded which confirms the expected molecular weight of the synthesized compounds. For compounds 3.11-3.16, the signals of positive ions $[\mathrm{M}+3]$ were additionally recorded, which characterizes the «isotopic profile» of sulfur [13].

${ }^{1} \mathrm{H}-\mathrm{NMR}$-spectra were characterized by the appearance of a low-field proton singlet of the exocyclic $\mathrm{NH}$-group of the quinazoline cycle at the $11.96-10.37 \mathrm{ppm}[11,12]$ and the amide $\mathrm{NH}$-proton at the $11.03-9.90 \mathrm{ppm}$, which in some cases $(3.13,3.18,3.19,3.22,3.23)$ did not appear due to rapid deuterium exchange or doubles due to hydrazide hydrazone tautomerism $(3.9,3.12)$. It is necessary to note that it was not possible to describe the multiplicity and characteristic chemical shifts of the aromatic protons of both quinazoline residue and the heterocyclic component of the molecule for hydrazides $3.11,3.13-3.16,3.18-3.21$, $3.23-3.25$, since they were observed as multiplets, which was a consequence of prototrophic tautomerism and their significant solubility in DMSO.

In the ${ }^{1} \mathrm{H}$ NMR spectra of compounds 3.1-3.6, the signals of protons of the alicyclic residue were registered in a
Scheme 1

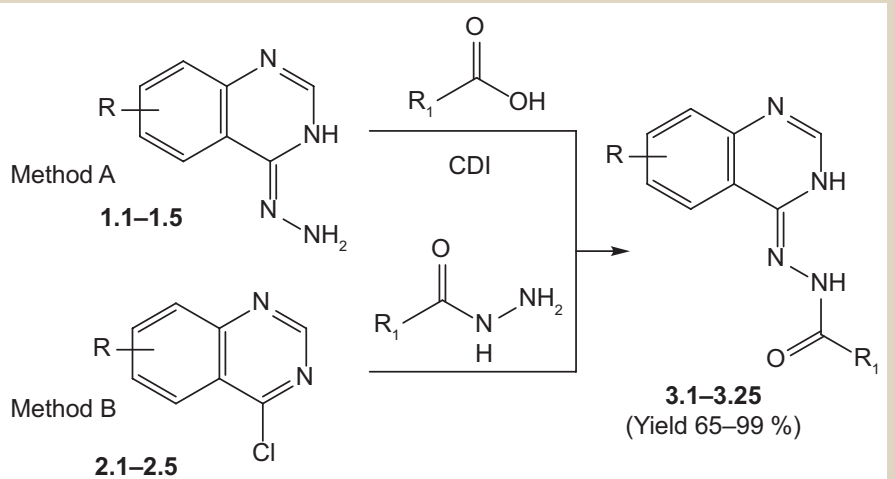

3.1 R = 8-F, $\mathrm{R}_{1}=$ cyclopropyl; $3.2 \mathrm{R}=6-\mathrm{Cl}, \mathrm{R}_{1}=$ cyclopropyl; $3.3 \mathrm{R}=\mathrm{H}, \mathrm{R}_{1}=$ adamantan -1-yl; 3.4 R = 8- $\mathrm{CH}_{3}, \mathrm{R}_{1}=$ adamantan-1-yl; 3.5 $\mathrm{R}=7-\mathrm{F}, \mathrm{R}_{1}=$ adamantan-1-yl; $3.6 \mathrm{R}=6-$ $\mathrm{Cl}, \mathrm{R}_{1}=$ adamantan-1-yl; $3.7 \mathrm{R}=8-\mathrm{CH}_{3}, \mathrm{R}_{1}=$ furan-3-yl; $3.8 \mathrm{R}=8-\mathrm{F}, \mathrm{R}_{1}=$ furan-3-yl; 3.9 $\mathrm{R}=6-\mathrm{Cl}, \mathrm{R}_{1}=$ furan-3-yl; $3.10 \mathrm{R}=6-\mathrm{Br}, \mathrm{R}_{1}=$ furan-3-yl; $3.11 \mathrm{R}=\mathrm{H}, \mathrm{R}_{1}=$ thiophen-2-yl; 3.12R = 7-F, $\mathrm{R}_{1}=$ thiophen-2-yl; $3.13 \mathrm{R}=8-\mathrm{CH}_{3}, \mathrm{R}_{1}=$ thiophen-3-yl; $3.14 \mathrm{R}=7-\mathrm{F}, \mathrm{R}_{1}=$ thiophen-3-yl; 3.15R $=6-\mathrm{Cl}, \mathrm{R}_{1}=$ thiophen-3-yl; 3.16 $\mathrm{R}=6-\mathrm{Br}, \mathrm{R}_{1}=$ thiophen-3-yl; $3.17 \mathrm{R}$ $=\mathrm{H}, \mathrm{R}_{1}=$ benzofuran-2-yl; $3.18 \mathrm{R}=8-\mathrm{CH}_{3}, \mathrm{R}_{1}$ = benzofuran-2-yl; 3.19 R = 7-F, $\mathrm{R}_{1}$ = benzofuran-2-yl; 3.20 R=6-Cl, $\mathrm{R}_{1}=$ benzofuran-2-yl; $3.21 \mathrm{R}=6-\mathrm{Br}, \mathrm{R}_{1}=$ benzofuran-2yl; 3.22 R =H, $\mathrm{R}_{1}=$ pyridin-2-yl; $3.23 \mathrm{R}=\mathrm{H}, \mathrm{R}_{1}=$ pyridin-3-yl; $3.24 \mathrm{R}=\mathrm{H}, \mathrm{R}_{1}=$ pyridin-4yl; $3.25 R=6-B r, R_{1}=$ pyridin-4-yl.

high field. In some cases mentioned signals were doubled, that was also caused by the tautomeric transitions. As for compounds 3.1 and 3.2 four protons of $2^{\text {nd }}$ and $3^{\text {rd }}$ position of the cyclopropyl moiety were registered as a multiplet at the $0.97-0.63 \mathrm{ppm}$, and the proton of $1^{\text {st }}$ position as a multiplet at 1.67-1.84 ppm. The splitting of the protons of the adamantan residue of compound 3.3 was interesting: they resonated as two six-proton doublets of «bridge» protons at the $1.75 \mathrm{ppm}\left(\mathrm{H}-4.4^{\prime}, \mathrm{H}-6.6^{\prime}, \mathrm{H}-10.10^{\prime}\right)$ and the $1.92 \mathrm{ppm}\left(\mathrm{H}-2,2^{\prime}, \mathrm{H}-8,8^{\prime}, \mathrm{H}-9,9^{\prime}\right)$, and three-proton singlet of «nodal» protons at the $2.06 \mathrm{ppm}(\mathrm{H}-3, \mathrm{H}-5, \mathrm{H}-7)$; for compounds 3.5 and 3.6 , the protons of the adamantan residue resonated as multiplets at $2.23-1.55 \mathrm{ppm}$. In the ${ }^{1} \mathrm{H}$ NMR spectra of compounds $3.4,3.7,3.13,3.18$, there was a three-proton singlet of the methyl group of the position 8 at the $2.49-2.53 \mathrm{ppm}$.

It is interesting to notice that the ${ }^{1} \mathrm{H}-\mathrm{NMR}$ spectrum of compound 3.17 made it possible to characterize both aromatic and heterocyclic protons. The aromatic protons of the quinazoline ring form the characteristic $A B C D$ system: $\mathrm{H}-5$ and $\mathrm{H}-8$ were observed as doublets at the 8.00 and the 7.80 ppm respectively, and $\mathrm{H}-6$ and $\mathrm{H}-7$ - triplets at the 7.57 and the $7.50 \mathrm{ppm}$ respectively $[11,12,14]$. As for proton $\mathrm{H}-2$ of quinazoline cycle, it resonated as a singlet at the $8.12 \mathrm{ppm}$, that is typical for most compounds of this series $[2,15]$. Protons of heteryl substituents had classical chemical shifts and multiplicity $[16,17]$.

As a result of the study of antimicrobial and antifungal activity, it was established (Table 1) that synthesized compounds in the concentration range of $200.0-12.5 \mu \mathrm{g} / \mathrm{ml}$ were active against the studied strains. The results' analysis of the effect of the test compounds on the growth of Staphylococcus aureus and Escherichia coli, showed that the greatest bacteriostatic $(12.5-50.0 \mu \mathrm{g} / \mathrm{ml})$ and bacterici- 
Table 1. Antimicrobial and antifungal activity of synthesized compounds*

\begin{tabular}{|c|c|c|c|c|c|c|c|c|}
\hline \multirow[t]{3}{*}{ Compound } & \multicolumn{8}{|c|}{ Investigated strains } \\
\hline & \multicolumn{2}{|c|}{ Escherichia coli } & \multicolumn{2}{|c|}{ Staphylococcus aureus } & \multicolumn{2}{|c|}{ Pseudomonas aeruginosa } & \multicolumn{2}{|c|}{ Candida albicans } \\
\hline & MIC $\mu \mathrm{g} / \mathrm{ml}$ & $\mathrm{MBC} \mu \mathrm{g} / \mathrm{ml}$ & $\mathrm{MIC} \mu \mathrm{g} / \mathrm{ml}$ & $\mathrm{MBC} \mu \mathrm{g} / \mathrm{ml}$ & MIC $\mu \mathrm{g} / \mathrm{ml}$ & $\mathrm{MBC} \mu \mathrm{g} / \mathrm{ml}$ & MIC $\mu \mathrm{g} / \mathrm{ml}$ & $\mathrm{MBC} \mu \mathrm{g} / \mathrm{ml}$ \\
\hline 3.1 & 100 & 200 & 100 & $>200$ & 50 & 100 & 50 & 50 \\
\hline 3.2 & 100 & 200 & 100 & 200 & 50 & 100 & 200 & 200 \\
\hline 3.3 & 200 & 200 & 50 & 200 & 100 & 100 & 50 & 100 \\
\hline 3.21 & 100 & 200 & 50 & 100 & 100 & 200 & 25 & 50 \\
\hline 3.22 & 50 & 100 & 12.5 & 50 & 50 & 100 & 50 & 100 \\
\hline 3.23 & 100 & 100 & 12.5 & 200 & 100 & 100 & 50 & 100 \\
\hline Nitrofural & 1.5 & - & 6.25 & - & 6.25 & - & 25.0 & - \\
\hline Trimethoprim & 50 & 50 & 31.2 & 62.5 & 62.5 & 125 & 62.5 & 125 \\
\hline Ketoconazole & - & - & - & - & - & - & 25 & - \\
\hline
\end{tabular}

*: the antimicrobial and antifungal activity of other synthesized compounds is MIC-100-200 $\mu \mathrm{g} / \mathrm{ml}, \mathrm{MBK}-100-200 \mu \mathrm{g} / \mathrm{ml}, \mathrm{MFK}-100-200 \mu \mathrm{g} / \mathrm{ml}$.

dal $(50-100 \mu \mathrm{g} / \mathrm{ml})$ effects were exhibited by substances 3.22 and 3.23 , which in hydrazide fragment contain a pyridine residue $(3.22,3.23)$. Towards to Pseudomonas aeruginosa, the investigated substances did not exhibit significant bacteriostatic and bactericidal activity (MIC-50-100 $\mu \mathrm{g} / \mathrm{ml}, \mathrm{MBC}-100-200 \mu \mathrm{g} / \mathrm{ml})$.

The compound 3.21 N'-(6-bromoquinazolin-4(3H)ylidene)benzofuryl-2-carbohydrazide (MIC $25 \mu \mathrm{g} / \mathrm{ml}$, MFC $50 \mathrm{ug} / \mathrm{ml}$ ) was comparable to the reference standard and exhibited the greatest antifungal activity. Interestingly, that compound 3.1 (N'-(7-fluoroquinazoline-4(3H)-ylidene) cyclopropanecarbohydrazide) did not show the activity against Staphylococcus aureus and Escherichia coli (100-200 $\mu \mathrm{g} / \mathrm{ml})$, but revealed bactericidal effect against Pseudomonas aeruginosa (MIC-50 $\mu \mathrm{g} / \mathrm{ml}, \mathrm{MBC}-100 \mu \mathrm{g} / \mathrm{ml}$ ). The study of the fungistatic and fungicidal action of this compound against Candida albicans showed that it exhibited high activity (MIC and MFC $50 \mu \mathrm{g} / \mathrm{ml}$ ). It is also necessary to mention the compounds 2.22 and 2.23, which exhibited fungicidal activity on Candida albicans (MIC $50 \mu \mathrm{g} / \mathrm{ml}$, MFC $100 \mu \mathrm{g} / \mathrm{ml}$ ).

So the studies of N'-[quinazoline-4(3H)-ylidene]-2(3) hydrazides antimicrobial and antifungal activity showed that the most active compounds were N'-[quinazolin-4 (3H)-ylidene]-2(3)-heterylcarbohydrazides.

\section{Conclusions}

1. Preparative methods for the synthesis of [quinazoline-4(3H)-ylidene]hydrazides of cycloalkyl-(hetaryl) carboxylic acids are considered. The choice of presented methods depends on order of receiving of the condensed heterocyclic and biologically active compounds.

2. The individuality and structure of the synthesized compounds was confirmed by elemental analysis, ${ }^{1} \mathrm{H}-\mathrm{NMR}$ and LC-MS spectra. It was shown, that prototropic (hydrazine-hydrazone) tautomerism is typical for synthesized compounds and, importantly, this tautomeric process leads to the changes in the integrated intensity and chemical shifts of both aromatic protons and protons of alicyclic groups.

3. The antimicrobial and antifungal activity of the new N'-[quinazoline-4 (3H)-ylidene]-2 (3)-cycloalkyl(hetaryl)-hydrazide derivatives was studied. It was shown, that N'-[quinazoline-4(3H)-ylidene]-2(3)-heterylcarbo-hydrazides exhibited the greatest antimicrobial activity, containing a pyridine moiety in the molecule, that exceeded the activity of the trimethoprim reference standard. The greatest anti- fungal activity was shown by N'-(6-bromoquinazolin-4(3H)ylidene)benzofuryl-2-carbohydrazide, that is comparable to the comparison standard ketoconazole.

Prospects for further research. During the next stage of our studies, it is planned to establish acute toxicity indicators, as well as to study the biological activity of synthesized compounds. In addition, the obtained compounds are prospective synthons in order to receive the tricyclic derivatives.

Фінансування: Аослідження виконане в рамках НАР Запорізького Аержавного медичного університету “Спрямований пошук біологічно активних речовин серед анельованих похідних хіназоліну та птериАину" № Аержреєстрації 0117U006961 (2017-2021).

Conflicts of Interest: authors have no conflict of interest to declare. Конфлікт інтересів: віАсутній.

Information about authors:

Schabelnyk K. P., PhD, Associate Professor of the Department of Pharmaceutical Chemistry, Zaporizhzhia State Medical University, Ukraine.

Kholodnyak S.V., PhD, Senior Lecturer of the Department of Organic and Bioorganic Chemistry, Zaporizhzhia State Medical University, Ukraine.

Berest G.G., PhD, Associate Professor of the Department of Pharmacognosy, Pharmaceutical Chemistry and Medicinal Preparations Technology of FPE, Zaporizhzhia State Medical University, Ukraine.

Kovalenko S. I., Drhab, Professor, Head of the Department of Organic and Bioorganic Chemistry, Zaporizhzhia State Medical University, Ukraine.

Polishchuk N. M., MD, PhD, Senior Lecturer of the Department of Microbiology, Virology and Immunology, Zaporizhzhia state medical university, Ukraine.

Kamyshnyi O. M., MD, PhD, DSc, Professor, Head of the Department of Microbiology, Virology and Immunology, Zaporizhzhia state medical university, Ukraine.

\section{Відомості про авторів:}

Шабельник К. П., канА. фарм. наук, Аоцент

каф. фармацевтичної хімії, Запорізький державний медичний університет, Україна.

Холодняк С. В., канА. фарм. наук, старший викладач каф. органічної та біоорганічної хімії, Запорізький державний медичний університет, Україна.

Берест Г. Г., канА. фарм. наук, Аоцент каф. фармакогнозії, фармацевтичної хімії і технології ліків ФПО, Запорізький Аержавний медичний університет, Україна.

Коваленко С. І., А-р фарм. наук, професор, зав. каф. органічної та біоорганічної хімії, Запорізький Аержавний медичний університет, Україна. 
Поліщук Н. М., канА. меА. наук, старший викладач каф. мікробіології, вірусології та імунології, Запорізький Аержавний медичний університет, Україна. Камишний О. М., А-р меА. наук, професор, зав. каф. мікробіології, вірусології та імунології, Запорізький Аержавний медичний університет, Україна.

\section{Сведения об авторах:}

Шабельник К. П., канА. фарм. наук, Аоцент

каф. фармацевтической химии, Запорожский государственный медицинский университет, Украина.

ХолоАняк С. В., канА. фарм. наук, старший преподаватель каф. органической и биоорганической химии, Запорожский государственный медицинский университет, Украина. Берест Г. Г., канА. фарм. наук, Аоцент каф. фармакогнозии, фармацевтической химии и технологии лекарств ФПО, Запорожский госуАарственный меАицинский университет, Украина.

Коваленко С. И., А-р фарм. наук, профессор, зав. каф. органической и биоорганической химии, Запорожский государственный меАицинский университет, Украина.

Полищук Н. М., канА. меА. наук, старший преподаватель каф. микробиологии, вирусологии и иммунологии, Запорожский государственный медицинский университет, Украина.

Камышный А. М., А-р меА. наук, профессор,

зав. каф. микробиологии, вирусологии и иммунологии, Запорожский государственный меАицинский университет, Украина.

Наяійшла Ао реАакції / Received: 06.10.2017

Після Аоопрацювання / Revised: 07.11.2017

Прийнято Ао Аруку / Accepted: 08.12.2017

\section{References}

[1] (2012) World Health Statistics 2012: Monitoring health for the SDGs (2012) Retrieved from: http://www.who.int/gho/publications/world_ health_statistics/2012.

[2] Karpenko, O. V. (2007). Syntez anelovanykh heterotsyklichnykh spoluk pokhidnykh 4-hidrazynokhinazolinu ta yikh biolohichna aktyvnist (Avtoref. dis...kand. farm. nauk). [Synthesis of annelated heterocyclic compounds of 4-hydrasinoquinazoline and their biological activity]. (Extended abstract of candidate's thesis). Zaporizhzhia [in Ukrainian].

[3] Nesterova, N. O., Kovalenko, S. I., Karpenko, O. V., Bielienichev, I. F., Maksimov, Yu. M., Vrynchanu, N. O., \& Novik, L. V. (2004). Syntez i protymikrobna aktyvnist N-[(5-R-furan-2-il)-metylen]-N-[3-(5-R-furan2-il)aliliden]-N'-khinazolin-4-il-hidrazyniv. [Synthesis and antimicrobial activity of N-[(5-R-furan-2-yl)-methylene] and N-[3-(5-R-furan-2-yl) allylidene]-N'-quinazoline-4-yl-hydrazines]. Farmatsevtychnyj zhurnal, $6,79-83$ [in Ukrainian].

[4] loffe, B. V., Kuznecov M. A., \& Potekhin, A. A. (1979). Khimiya organicheskikh proizvodnykh gidrazina [Chemistry of organic derivatives of hydrazine]. Leningrad: Khimiya [in Russian].

[5] El-Hashash, M. A., Rizk, S. A., El-Bassiouny, F. A., \& Darwish, K. M. (2012) Reactivity of 2-ethoxyquinazolin-4-yl hydrazine and its Use in Synthesis of Novel Quinazoline Derivatives of Antimicrobial Activity. Global Journal of Health Science. 4(1), 174-183. doi: 10.5539/gihs.v4n1p174.

[6] Claesen M., \& Wanderhaeghe M. (1959) Derives de quinazoline. Bull. Soc. Chim. Belg., 68, 220-222.

[7] Volianskyi, Yu. L., Hrytsenko, I. S., Shyrobokov, V. P., et al. (2004) Vyvchennia spetsyfichnoi aktyvnosti protymikrobnykh likarskykh zasobiv [Specific activity studying of antimicrobial drugs]. Kyiv. [in Ukrainian].

[8] Nakaz Ministerstva okhorony zdorovia Ukrainy «Pro zatverdzhennia metodychnykh vkazivok «Vyznachennia chutlyvosti mikroorhanizmiv do antybakterialnykh preparativ» vid 05.04.2007 roku №167 [Order of the Ministry of Health of Ukraine On approval of guidance «Determination of the sensitivity of microorganisms to antibiotics» from April, 05, 2007, №167]. [in Ukrainian].

[9] Wayne, P. A. Performance Standards for Antimicrobial Disk Susceptibility Tests; Approved Standard. Clinical and Laboratory Standards Institute. CLSI M2-A9.

[10] Gizatullina, E'. M., \& Karcev, V. G. (1993). Annelirovanie 1,2,4-triazol'nogo yadra na osnove a-gidrazinozameshchennykh geterociklov $\mathrm{i}$ ikh gidrazonov [Annelation of 1,2,4-triazole nucleus on the basis of a-hydrazino-substituted heterocycles and their hydrazones]. Khimiya geterociklicheskikh soedinenij, 12, 1587-1613 [in Russian].
[11] Karpenko, O. V, \& Kovalenko, S. I. (2005). Syntez 2-R-tryazolo[1,5-s] khinazoliniv. Povidomlennia 1. [Synthesis of 2-R-triazolo [1,5-c] quinazoline. Message 1]. Zhurnal orhanichnoi ta farmatsevtychnoi khimii, 3, 2(10), 47-54 [in Ukrainian].

[12] Karpenko, O. V., \& Kovalenko, S. I. (2005). Syntez 2-R-tryazolo[1,5-s] khinazoliniv. Povidomlennia 2. [Synthesis of 2-R-triazolo [1,5-c] quinazoline. Message 2]. Zhurnal orhanichnoi ta farmatsevtychnoi khimiyi, 3, 4(12), 61-69 [in Ukrainian].

[13] Zaikin, V. G., Varlamov, A. V., Mikaya, A. I., \& Prostakov, N. S. (2001). Osnovy mass-spektrometrii organicheskikh soedinenij [Fundamentals of mass spectrometry of organic compounds]. Moscow: Nauka, Interperiodika [in Russian].

[14] Karpenko, O. V, \& Kovalenko, S. I. (2006). Syntez 2-R-tryazolo[1,5-s] khinazoliniv. Povidomlennia 3. [Synthesis of 2-R-triazolo [1,5-c]quinazoline. Message 3]. Zhurnal orhanichnoi ta farmatsevtychnoi khimiyi, 4, 2(14), 65-70 [in Ukrainian].

[15] Voloshyna, V. O. (2011). Syntez, fizyko-khimichni ta biolohichni vlastyvosti zamishchenykh 1,2,4-tryazolu ta yoho kondensovanykh pokhidnykh (Avtoref. dis...kand. farm. nauk) [Synthesis, physico-chemical and biological properties of 1,2,4-triazole substituted and its fused derivatives]. (Extended abstract of candidate's thesis). Zaporizhzhia [in Ukrainian].

[16] Khausser, K. Kh., \& Kal'bitcer, Kh. R. (1993). YAMR v medicine ibiologii: struktura molekul, tomografiya, spektroskopiya in vivo [NMR in medicine and biology: molecular structure, tomography, in vivo spectroscopy]. Kyiv: Nauk. dumka [in Russian].

[17] E'rnst R., Bodenkhauzen, Dzh., \& Vokaun, A. (1990). YAMR v odnom $i$ dvukh izmereniyakh [NMR in one and two dimensions]. Moscow: Mir [in Russian]. 